\title{
HIGHLIGHTS
}

GENETICS

\section{HER3 overexpression in breast cancer conveys a poor prognosis}

HER2 testing and targeted therapy are well established in the management of breast cancer. Now, a study by Chiu and colleagues reports that HER3 status is also an important prognostic marker of breast cancer-specific survival.

\section{4 ...HER3 expression was...} significantly associated with decreased disease-specific survival 77

HER2 cellular signaling is affected by the expression of other type 1 growth factor receptor family members (T1GFR), notably HER3. Moreover, "HER2:HER3 heterodimers represent the most mitogenic couplet among all T1GFR dimers or heterodimers," explains lead investigator Sam Wiseman. "Thus, the investigation of HER3 as a prognostic marker in invasive breast cancer appeared a highly promising and clinically

applicable candidate."

Tumor samples from 4,046 patients with invasive breast carcinoma were assessed for expression of T1GFR family members HER1, HER2, HER3 and HER4. In 10\% of these tumors, HER3 overexpression was identified and was significantly associated with decreased disease-specific survival.

Furthermore, in tumors with normal HER1 and HER2 expression, HER3 conveyed a negative prognostic effect on breast cancer-specific survival. "HER3 may represent a target for anticancer therapy in this patient subset that otherwise do not express molecules targeted by current molecular treatments," Sam Wiseman concludes.

Lisa Richards

Original article Chiu, C. G. et al. HER-3 overexpression is prognostic of reduced breast cancer survival: a study of 4,046 patients. Ann. Surg. 251, 1107-1116 (2010) 